\title{
Estrategias de manejo de conflicto en clave emocional
}

\author{
Carlos Montes ${ }^{*}$, Dámaso Rodríguez ${ }^{2}$ y Gonzalo Serrano ${ }^{2}$
}

1 Escuela Universitaria de Relaciones Laborales de Lugo. Universidad de Santiago de Compostela (España)

2 Facultad de Psicología. Universidad de Santiago de Compostela (España)

\begin{abstract}
Resumen: Un conflicto es un proceso dinámico y complejo que se alimenta de emociones. Por lo tanto, para entender los procesos de gestión de conflicto, es necesario estudiar la influencia de las variables emocionales que rodean las disputas. El propósito de este artículo es explorar el modo en que los estados de ánimo de las personas pueden orientar sus estrategias de manejo de conflictos. A diferencia de otros trabajos que manipulan artificialmente las emociones, este estudio se centra en las diferencias de la experiencia emocional de las personas que se producen de modo natural. Los resultados sugieren que los estados de ánimo influyen sobre la elección de las estrategias de gestión de conflicto. Concretamente, se ha hallado que el afecto positivo predice la estrategia de integración, mientras que el afecto negativo predice la estrategia de dominación. Como conclusión se apunta que la influencia intrapersonal de las emociones es especialmente notoria cuando las personas experimentan estados de elevada activación emocional. Palabras clave: Conflicto; emociones; estado de ánimo; estrategias de manejo de conflictos.
\end{abstract}

\section{Introducción}

El conflicto es un proceso básico e inevitable que caracteriza la dinámica interna de las relaciones humanas. Surge cuando dos o más personas perciben una oposición de metas, objetivos y/o valores y ven a la otra parte como una interferencia para satisfacer sus deseos (Putnam y Poole, 1987, p. 552). Así, la vida cotidiana ofrece múltiples y variados ejemplos de conflictos como, por ejemplo, un inquilino que deja de pagar el alquiler porque no funciona correctamente la calefacción, una pareja que se separa debido a irreconciliables desavenencias conyugales, o una oficina en la que sólo se dispone de una fotocopiadora y dos trabajadores necesitan utilizarla al mismo tiempo. En otras palabras, el conflicto es una expresión natural del comportamiento de las personas, que subyace latente a cualquier forma de interacción social (Rahim, 1986).

Con el fin de resolver la disputa y alcanzar algún tipo de acuerdo, las partes despliegan diferentes estrategias y tácticas. Mientras las estrategias incorporan objetivos definidos que orientan y organizan el enfoque general desde el que se trata de resolver el conflicto, las tácticas representan una implementación de las estrategias en términos de conductas específicas (Pruitt y Carnevale, 1993). De acuerdo con la perspectiva tradicional, las partes en conflicto realizan un análisis racional de los posibles resultados de cada estrategia y/o táctica antes de efectuar una elección de entre su repertorio conductual e iniciar un curso de acción. Sin embargo, esta visión normativa ha ido cambiando progresivamente, gracias a la acumulación de evidencias empíricas que dan

* Dirección para correspondencia [Correspondence address]:

Carlos Montes. Universidad de Santiago de Compostela, Escuela Universitaria de Relaciones Laborales, Complejo Docente - Campus Universitario de Lugo, 27002, Lugo (España).

E-mail: carlos.montes@,usc.es
Title: Conflict handling strategies in emotional key.

Abstract: Conflict is a dynamic and complex process emotionally actived. Therefore, in order to understand conflict management processes, it is necessary to study the influence of emotional variables which surround confrontations. The purpose of this paper is to explore how mood can influence the choice of conflict management strategies. Unlike other studies that artificially manipulate emotions, this one focuses on the differences in emotional experience that occur naturally. Results suggest that moods influence the choice of conflict handling strategies. Specifically, it has been found that positive affect predicts integrating, while negative affect predicts dominating. In conclusion, it is suggested that the intrapersonal influence of emotions is particularly noticeable when people experience high-aroused affective states.

Key words: Conflict; emotions; mood; conflict management strategies.

apoyo a la tesis de que los sujetos hacen frente a un conflicto influidos por su estado emocional (Barry y Fulmer, 2004). No en vano, la propia experiencia de un conflicto en una intensa fuente de emociones (Weingart, Bear, y Todorova, 2009), y éstas influyen, a su vez, sobre el comportamiento de las partes en el desarrollo del propio conflicto (Barry, Fulmer, y Van Kleef, 2004), determinando tanto el contenido de las propuestas realizadas como el modo en que se responde a las propuestas recibidas.

A pesar del creciente número de investigaciones que han estudiado la incidencia de las variables emocionales en los procesos de gestión de disputas, aún se desconocen gran parte de los mecanismos afectivos que operan detrás del manejo de conflictos (Nair, 2008). El presente trabajo trata de rellenar, al menos en parte, estas lagunas. Concretamente, intenta ampliar nuestro conocimiento mediante la comprensión de las claves emocionales que explican la preferencia por las estrategias de gestión de conflictos. A diferencia de investigaciones previas (p.e., Carnevale e Isen, 1986), donde se inducen artificialmente diferentes estados de ánimo y/o emociones, en esta investigación se defiende que los estilos de manejo de conflictos derivan incluso de la experiencia afectiva natural o incidental de los sujetos; esto es, no inducida en laboratorio. La razón de este planteamiento estriba en que se ha hallado que la influencia de los estados emocionales, por leves que sean, puede perdurar incluso más que la propia experiencia emocional (Andrade y Ariely, 2009; Forgas y George, 2001).

\section{Estrategias y tácticas de manejo de conflictos}

Como puede imaginarse, existen infinidad de estrategias y tácticas para hacer frente a un conflicto. No obstante hay un acuerdo extendido en aceptar como sistema conceptual de clasificación el Dual Concern Model o Modelo de Intereses 
Dobles, propuesto originalmente por Blake y Mouton (1964) y posteriormente adoptado y reinterpretado por muchos otros (Hall, 1969; Pruitt, 1983; Pruitt y Rubin, 1986; Rahim y Bonoma, 1979; 'Thomas, 1976).

El Dual Concern Model especifica que el modo en que una persona responde a un conflicto depende de su orientación motivacional y, en este sentido, defiende la existencia de dos motivos básicos: El interés propio y el interés por los demás. La fuerza de cada una de estas dimensiones motivacionales puede variar en un continuo alto-bajo, lo que, finalmente, conduce a cinco estrategias de gestión de conflictos diferentes (Rahim, 1983): (a) integración (alto interés propio y alto interés por los demás); (b) dominación (alto interés propio y bajo interés por los demás); (c) servilismo (bajo interés propio y alto interés por los demás); (d) evitación (bajo interés propio y bajo interés por los demás); y (e) compromiso (moderado interés propio y moderado interés por los demás). En la Tabla 1 se recoge una descripción más detallada de cada estrategia de manejo de conflictos.

Tabla 1. Estrategias y tácticas de manejo de conflictos.

\begin{tabular}{|c|c|c|}
\hline Estrategia & Objetivo principal & Tácticas \\
\hline Integración & $\begin{array}{l}\text { Encontrar soluciones sa- } \\
\text { tisfactorias para ambas } \\
\text { partes }\end{array}$ & $\begin{array}{l}\text { Plantear alternativas; abrir líneas de comunicación; hacer declaraciones descriptivas, abier- } \\
\text { tas, calificativas, solicitantes o de apoyo; hacer concesiones; aceptar la responsabilidad; } \\
\text { maximizar las semejanzas y minimizar las diferencias. }\end{array}$ \\
\hline Dominación & $\begin{array}{l}\text { Encontrar un acuerdo sa- } \\
\text { tisfactorio a nivel indivi- } \\
\text { dual }\end{array}$ & $\begin{array}{l}\text { Usar poder de posición, agresión, dominación verbal, perseverancia; hacer declaraciones } \\
\text { de confrontación, acusaciones, críticas personales, rechazo, amenazas; ser sarcástico; ha- } \\
\text { cer burlas, preguntas agresivas; negar la responsabilidad a expensas de la otra parte. }\end{array}$ \\
\hline Servilismo & $\begin{array}{l}\text { Ceder a los deseos de la } \\
\text { otra parte }\end{array}$ & $\begin{array}{l}\text { Emplear conductas de complacencia; aceptar pasivamente las decisiones de los demás; } \\
\text { hacer declaraciones concesivas, negar o fracasar al expresar las propias necesidades. }\end{array}$ \\
\hline Evitación & $\begin{array}{l}\text { Intentar sortear el } \\
\text { desacuerdo }\end{array}$ & $\begin{array}{l}\text { Abandonar física y/o psicológicamente el conflicto; negar la existencia del conflicto; } \\
\text { cambiar y/o evitar determinados temas; emplear declaraciones no-comprometidas; hacer } \\
\text { declaraciones irrelevantes o bromear. }\end{array}$ \\
\hline Compromiso & $\begin{array}{l}\text { Proponer una solución } \\
\text { intermedia }\end{array}$ & $\begin{array}{l}\text { Reducir las diferencias; buscar un camino intermedio con la otra parte; sugerir un inter- } \\
\text { cambio de ofertas; maximizar las ganancias y minimizar las perdidas; ofrecer una resolu- } \\
\text { ción rápida al conflicto. }\end{array}$ \\
\hline
\end{tabular}

Fuente: Elaboración propia a partir de Hocker y Wilmot (1998), Montes, Serrano y Rodríguez (2010) y Rahim (2002).

La investigación ha dado sólido apoyo empírico a esta taxonomía de cinco estrategias. Por ejemplo, diferentes estudios observacionales (p.e., Carnevale y Lawler, 1986; De Dreu, Giebels y Van de Vliert, 1998; Weingart, Hyder y Prietula, 1996), a partir del sistema de codificación desarrollado por Pruitt y Lewis (1975), han hallado que la dominación se representa mediante el compromiso con la propia posición y el empleo de argumentos persuasivos. Asimismo, han encontrado que la integración se caracteriza por el intercambio de información y manifestaciones abiertas de cooperación. Otros trabajos empleando cuestionarios han convergido en la misma taxonomía de cinco categorías (p.e., De Dreu, Evers, Beersma, Kluwer y Nauta, 2001; Rahim y Magner, 1995).

Estas estrategias se pueden organizar de acuerdo con las dimensiones integrativas y distributivas de resolución de conflictos (Rahim, 2002). Continuando con la interpretación del Dual Concern Model (ver Figura 1), la dimensión integrativa representaría el interés (alto-bajo) de la persona por uno mismo y los demás, siendo la integración y la evitación los extremos de dicha dimensión. La dimensión distributiva, por su parte, representaría el interés (alto-bajo) de la persona por uno mismo o los demás, de modo que la dominación estaría en un polo y el servilismo en el opuesto. Finalmente, el compromiso se encuadraría en un punto intermedio en ambas dimensiones ya que, si bien se centra en los intereses de ambas partes, lo hace de modo moderado y no los satisface completamente.

En resumen, podemos señalar que la investigación sobre estrategias y tácticas de negociación apunta a la existencia de un repertorio estratégico -integración, distribución, evitación, servilismo y compromiso-, al que subyace una dimensión distributiva y otra integrativa (De Dreu, Beersma, Steinel y Van Kleef, 2007).

\section{Experiencia emocional y estrategias de manejo de conflicto}

A pesar de que no hace mucho tiempo que el estudio de las emociones estaba relegado a un segundo plano en la investigación científica sobre conflicto y negociación (Bazerman, Curhan, Moore y Valley, 2000), los últimos 30 años han sido testigo de una verdadera revolución emocional en la que el número de investigaciones y estudios sobre el impacto de las emociones en los procesos de resolución de conflictos ha aumentado ostensiblemente (Barsade y Gibson, 2007).

En aras de una mayor precisión conceptual, la literatura especializada suele hacer una distinción entre los diferentes términos que aluden a la experiencia emocional, a saber: estados de ánimo y emociones. Cada uno de estos conceptos afectivos difiere en términos de su duración, los cambios fisiológicos que lleva asociados y la intencionalidad que los ac- 
tiva (Frijda, 1993). Así, los estados de ánimo son más duraderos, de menor intensidad, y no se asocian con un objeto específico. Las emociones, en cambio, son discretas, de ma- yor intensidad, de menor duración y se dirigen hacia una persona, objeto o situación específica.

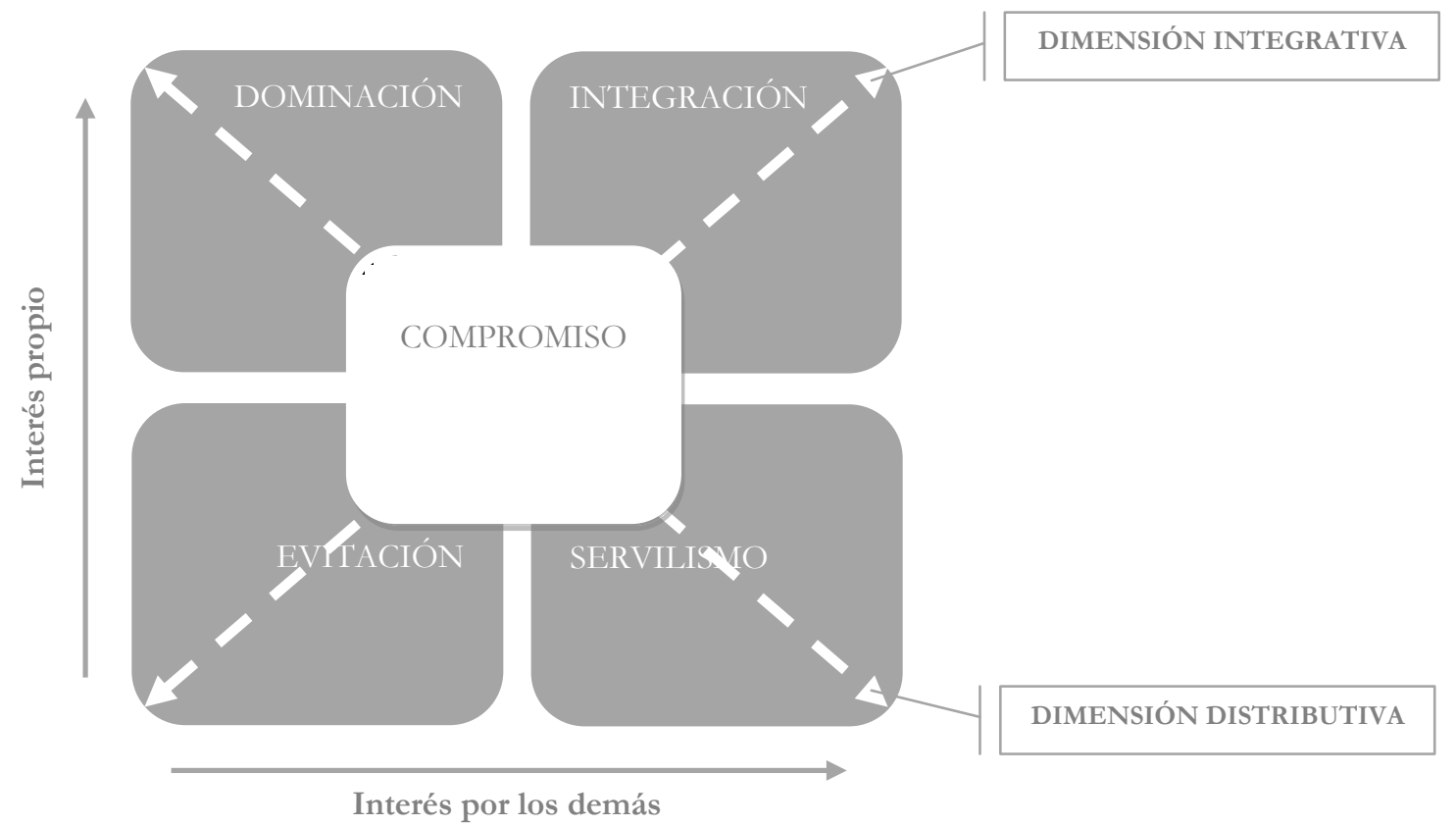

Figura 1. Dimensiones integrativa y distributiva de las estrategias de manejo de conflictos. (Fuente: Adaptado de Rahim, 2002, p. 221).

Lejos de ser baladí, esta distinción conceptual ha propiciado dos líneas paralelas y complementarias de investigación sobre el papel de la experiencia afectiva en la resolución de conflictos (Morris y Keltner, 2000). Por una parte, los estudios sobre el efecto intrapersonal de la experiencia emocional se centran en el impacto del afecto sobre el propio comportamiento de conflicto. Para ello estudian la capacidad predictiva de los estados de ánimo sobre el comportamiento de conflicto, generalmente mediante la inducción de estados de ánimo (p.e., Carnevale e Isen, 1986). Por su parte, los estudios sobre el efecto interpersonal de la experiencia emocional abordan el impacto del afecto sobre el comportamiento de los demás. Concretamente, estudian el impacto de las emociones discretas de una de las partes del conflicto sobre la conducta de la otra parte (p.e., Van Kleef, De Dreu y Manstead, 2004a; 2004b; Van Kleef, De Dreu, Pietroni y Manstead, 2006).

El presente trabajo se integra dentro de la primera línea de investigación, esto es, el modo en que el propio estado de ánimo puede afectar a las estrategias que se despliegan para manejar un conflicto. En este sentido, existe un volumen considerable de investigaciones que demuestran que los individuos que experimentan emociones y estados de ánimo positivos tienden a comportarse de modo más cooperativo y conciliatorio (Allred, Mallozzi, Matsui y Raia, 1997; Baron, 1990; Baron, Fortin, Frei, Hauver y Shack, 1990; Carnevale e Isen, 1986; Forgas, 1998; Isen et al., 1987; Kramer, Newton y Pommerenke, 1993), mientras que aquellos sujetos que experimentan emociones o estados de ánimo negativos tienden a ser más competitivos (Allred et al., 1997; Baron et al., 1990; Forgas, 1998; Forgas y Cromer, 2004; Pillutla y Murnighan, 1996).

A pesar de ello, aún son pocos los trabajos que han explorado explícitamente el impacto de los estados de ánimo sobre la aproximación estratégica a un conflicto. Por ejemplo, dentro de un estudio sobre las preferencias por las estrategias de gestión de conflictos dentro de los equipos de trabajo, Desivilya y Yagil (2005) encontraron que las estrategias integrativas se relacionan con estados emocionales positivos, las estrategias distributivas se relacionan tanto con estados emocionales positivos como negativos, y que la estrategia de evitación sólo se relaciona con el estado de ánimo negativo. Rhoades, Arnold y Jay (2001), en su estudio sobre el impacto de los rasgos y estados afectivos sobre las motivaciones y conductas de los empleados durante episodios de conflicto, muestran que el afecto positivo se asocia a comportamientos integrativos y que, en cambio, el afecto negativo estimula la competitividad y el interés propio. Por su parte, Bell y Song (2005) en su trabajo sobre el papel de un rango de emociones discretas sobre las estrategias de conflicto, hallaron que el constructo emocional de "positividad relacional" -que engloba sentimientos positivos y de preocupación por la otra parte como, por ejemplo, respeto, agradecimiento, admira- 
ción, amigabilidad o comprensión-, se relaciona con la integración, el compromiso y el servilismo.

\section{El presente estudio}

Basándose en la literatura existente, el presente trabajo examina las relaciones entre los estados de ánimo naturales (no inducidos artificialmente) y la preferencia por las estrategias de gestión de conflictos. Concretamente, este estudio exploratorio examina si el grado en que una persona experimenta estados de ánimo positivos se relaciona más íntimamente con la dimensión integrativa de gestión de conflictos (especialmente con la estrategia integrativa), y si el grado en que una persona siente estados de ánimo negativos se relaciona más con la dimensión distributiva (especialmente con la estrategia de dominación).

A pesar de que estas hipótesis parecen ofrecer una visión polar de las emociones (negativas frente a positivas), existe amplia evidencia empírica que apoya que el afecto negativo y el afecto positivo son constructos independientes, en lugar de los extremos separados de un mismo continuo (Feldman y Russell, 1998; Watson y Tellegen, 1985). Así, puntuaciones elevadas en cada dimensión representan un estado de alta afectividad, mientras que puntuaciones bajas caracterizarían la ausencia relativa de implicación afectiva (Watson y Tellegen, 1985). Esto es, el afecto positivo, en su polo alto, se caracteriza por un estado de entusiasmo, activación y euforia (Watson, Clark y Tellegen, 1988), mientras que un bajo afecto positivo describe un estado de abatimiento, cansancio o somnolencia (Watson y Clark, 1997). Por su parte, un alto afecto negativo se caracteriza por un estado emocional de angustia, irritación o nerviosismo, frente a un bajo afecto negativo que se define como un estado de relajación, calma y serenidad (Watson et al., 1988).

Es por esto que, al igual que se ha señalado en otras investigaciones (p.e., Barry, 2008; Montes, Rodríguez y Serrano, 2012), se espera que la preferencia por las estrategias de manejo de conflictos pueda predecirse mejor a partir del perfil emocional completo de los sujetos, incluyendo paralelamente medidas de emociones y estados de ánimo positivos y negativos. De modo que también se hipotetiza que por cada estrategia de resolución de conflictos exista un perfil diferente de afecto positivo-afecto negativo.

\section{Método}

\section{Participantes}

Un total de 440 estudiantes (336 mujeres y 101 hombres, 3 participantes no especificaron su sexo) de la Universidad de Santiago de Compostela (USC) han participado voluntariamente en este estudio. Las edades de los sujetos comprendieron un rango de 17 a 48 años, con una media de edad de 20.7 años $(D T=3.8)$.

\section{Instrumentos}

Estados de ánimo: se ha empleado la escala PANAS (Watson et al., 1988), formada por 20 ítems que miden estados de ánimo experimentados subjetivamente. En esta escala los sujetos evaluaron el grado en que, de forma general, experimentan cada sentimiento o emoción, sobre una escala Likert de 1 ("nada o casi nada") a 5 ("muchísimo"). Diez ítems miden afecto positivo (AP), es decir, el grado en que una persona se siente entusiasmada, activa, alerta o enérgica. Los diez ítems restantes miden afecto negativo (AN) o, en otras palabras, estados emocionales de distrés subjetivo como el disgusto, el nerviosismo, la culpa o la tensión. En la versión original, Watson et al. (1988) encontraron una fiabilidad alfa de Cronbach de .88 y .87 para AP y AN, respectivamente. La versión española (Sandín et al., 1999) confirma la bidimensionalidad de la escala y la consistencia interna de la misma (entre .87 y .89 , para AP y entre .89 y .91 para AN).

Estrategias de gestión de conflictos: se han medido mediante el ROCI-II (Rahim, 1983), que admite tres formas de abordaje a los conflictos atendiendo al nivel jerárquico de la otra parte: conflictos con superiores (forma A), conflictos con subordinados (forma B), o conflictos con compañeros (forma C). Concretamente, se ha empleado la forma $\mathrm{C}$ que, a partir de 28 ítems, permite evaluar cinco estilos de gestión de conflictos -integración (7 ítems), evitación (6 ítems), dominación (5 ítems), servilismo (6 ítems) y compromiso (4 ítems)-, en una escala de respuesta tipo Likert de 5 puntos, de 1 ("completamente en desacuerdo") a 5 ("completamente de acuerdo"). Munduate, Ganaza y Alcaide (1993) validaron la escala en nuestro contexto socio-cultural, verificando la existencia de cinco estilos de manejo de conflictos independientes, cuya consistencia interna osciló entre .62 y .77, para los estilos compromiso e integración, respectivamente.

\section{Procedimiento}

Los participantes fueron reclutados en diferentes facultades de la Universidad de Santiago de Compostela (USC) por los autores del trabajo y miembros de su grupo de investigación, para colaborar en un estudio sobre los determinantes individuales de la gestión de conflictos. Una vez que prestaron su consentimiento, respondieron un cuadernillo que contenía las escalas. La administración fue colectiva y en horario regular de clase. En todo momento se garantizó la confidencialidad y el anonimato de los participantes. Completado el cuadernillo, se les agradeció su participación y se les informó del objetivo de la investigación.

\section{Análisis de datos}

Para contrastar si el estado de ánimo predice los estilos de gestión de conflicto (integración, dominación, servilismo, evitación y compromiso) se realizaron diferentes análisis de regresión -uno por cada estilo-, con el afecto positivo y negativo introducidos simultáneamente como predictores. Para 
garantizar la validez del procedimiento se analizaron los supuestos de normalidad, homocedasticidad y colinealidad. Todos los análisis fueron realizados con el paquete estadístico SPSS 17.0.

\section{Resultados}

La Tabla 2 recoge los estadísticos descriptivos para el afecto positivo, el afecto negativo y los cinco estilos de gestión de conflictos.

Tabla 2. Estadísticos descriptivos y correlaciones entre las variables de interés $(N=440)$.

\begin{tabular}{|c|c|c|c|c|c|c|c|c|c|c|c|}
\hline Variable & Media & $D T$ & Mín. & Máx. & 1 & 2 & 3 & 4 & 5 & 6 & 7 \\
\hline 1. AP & 3.05 & 0.65 & 1.00 & 4.60 & $(.84)$ & & & & & & \\
\hline 2. $\mathrm{AN}$ & 1.48 & 0.58 & 1.00 & 3.90 & -.09 & $(.86)$ & & & & & \\
\hline 3. Integración & 4.02 & 0.52 & 1.86 & 5.00 & $.23^{* * *}$ & -.06 & $(.86)$ & & & & \\
\hline 4. Evitación & 3.41 & 0.65 & 1.50 & 5.00 & .03 & .04 & $.23 * * *$ & $(.74)$ & & & \\
\hline 5. Dominación & 3.17 & 0.72 & 1.00 & 5.00 & $.14^{* * *}$ & .08 & $-.14^{* *}$ & -.01 & $(.77)$ & & \\
\hline 6. Servilismo & 2.98 & 0.62 & 1.33 & 4.83 & .08 & .06 & $.26 * * *$ & $.42^{* * *}$ & -.08 & $(.76)$ & \\
\hline 7. Compromiso & 3.93 & 0.44 & 2.00 & 5.00 & $.15^{* *}$ & -.04 & $.69 * * *$ & $.30 * * *$ & $-.12^{* *}$ & $.32^{* * * *}$ & $(.67)$ \\
\hline
\end{tabular}

Nota. En la diagonal se recoge la consistencia interna de las escalas.

$\mathrm{AP}=$ Afecto Positivo; $\mathrm{AN}=$ Afecto Negativo.

$* p<.05 ; * * p<.01 ; * * * p<.001$.

En primer lugar, cabe señalar que la consistencia interna de las escalas es satisfactoria (Nunnally, 1978). Asimismo, el análisis preliminar de correlaciones muestra que el afecto positivo y el afecto negativo no están relacionados, $r(440)=$ $.09, n s$, validando la independencia de los predictores. Por último, los resultados también muestran que el compromiso está íntimamente relacionado con la integración, $r(440=.69$, $p<.001$. Esto es, aparentemente el compromiso representaría una forma débil de integración (Montes et al., 2010; Pruitt y Rubin, 1986; Van de Vliert y Hordijk, 1989) y, por lo tanto, ha sido excluido de los análisis posteriores.

Antes de proceder con los análisis de regresión, se verificaron los supuestos de validación de los respectivos modelos. En primer lugar, se comprobó la posible existencia de colinealidad entre las variables contempladas en el estudio y, de acuerdo a los valores de tolerancia y de los factores de inflación de la varianza (FIV), se puede asumir que no existió riesgo significativo de colinealidad (Cohen, Cohen, West y
Aiken, 2003). Además, el estadístico Durbin-Watson permitió asumir que los residuos son independientes al estar los valores de los modelos de regresión dentro del rango 1.5-2.5 recomendado (George y Mallery, 2003). Por otro lado, el diagrama de dispersión de los pronósticos tipificados por los residuos tipificados mostró la igualdad de varianzas. Asimismo, el histograma de los residuos tipificados mostró una distribución normal de la varianza de los criterios. En resumen, las comprobaciones realizadas ponen de manifiesto la validez de los modelos $\mathrm{y}$, por lo tanto, se puede proceder a interpretar los resultados encontrados.

Con el objetivo de establecer en qué medida el estado de ánimo puede explicar la preferencia por los estilos de manejo de conflictos, se ha realizado un análisis de regresión para cada una de las estrategias de conflicto, empleando como variables predictoras el afecto positivo y el afecto negativo. Los resultados se presentan en la Tabla 3 .

Tabla 3. Regresión del estado de ánimo sobre los estilos de gestión de conflictos $(N=440)$.

\begin{tabular}{|c|c|c|c|c|c|}
\hline Estilo de Gestión de Conflictos & $B$ & $S E B$ & $\beta$ & $R^{2}$ & Durbin-Watson \\
\hline \multicolumn{6}{|l|}{ Integración } \\
\hline Afecto Positivo & .179 & .037 & $.226^{* * *}$ & $.054 * * *$ & 2.04 \\
\hline Afecto Negativo & -.033 & .042 & -.037 & & \\
\hline \multicolumn{6}{|l|}{ Evitación } \\
\hline Afecto Positivo & .028 & .048 & .028 & .002 & 1.91 \\
\hline Afecto Negativo & .042 & .054 & .038 & & \\
\hline \multicolumn{6}{|l|}{ Dominación } \\
\hline Afecto Positivo & .162 & .052 & $.146^{* *}$ & $.028 * *$ & 2.05 \\
\hline Afecto Negativo & .116 & .059 & $.093^{*}$ & & \\
\hline \multicolumn{6}{|l|}{ Servilismo } \\
\hline Afecto Positivo & .083 & .045 & .088 & .011 & 1.92 \\
\hline Afecto Negativo & .066 & .051 & .062 & & \\
\hline
\end{tabular}


El primer modelo de regresión, que evalúa la capacidad predictiva del AP y el AN sobre la integración, muestra que el afecto positivo, pero no el negativo, es un predictor significativo, $F(2,437)=12.45, p<.001$. Así, cuando los sujetos sienten emociones positivas tienden a mostrarse más colaboradores y procuran satisfacer tanto sus propios intereses como los de su oponente. El segundo modelo, en el que la variable dependiente es la evitación, no fue significativo, $F(2$, $437)=0.44, n s$. Por lo tanto, ni el AP ni el AN predicen significativamente la preferencia por dicha estrategia gestión de conflictos. Cuando el criterio es la estrategia de dominación, los resultados indican que tanto el AP como el AN son predictores significativos, $F(2,437)=6.19, p<.01$. De este modo, cuando los sujetos experimentan al mismo tiempo alto $\mathrm{AP}$ y alto AN tienen mayor preferencia por estrategias que maximicen su propio beneficio a expensas de los resultados del oponente. Con respecto al servilismo, los resultados señalan que, al igual que sucedía con la evitación, ni el AP ni el AN predicen significativamente la preferencia por dicha estrategia, $F(2,437)=2.36$, ns. Los coeficientes estandarizados $(\beta)$ recogidos en la Tabla 3 indican que las relaciones encontradas siguen las direcciones descritas en investigaciones previas (p.e., Desivilya y Yagil, 2005).

\section{Análisis adicionales}

Con el objetivo de profundizar en la relación entre las variables emocionales y las estrategias de manejo de conflictos, se llevó a cabo un análisis adicional basado en las dimensiones integrativa y distributiva subyacentes a las estrategias de manejo de conflictos (Thomas, 1976). Para realizarlo se calcularon dos índices diferentes, correspondientes a ambas dimensiones, a partir de las puntuaciones de los sujetos en el ROCI-II (ver Rahim, 2002, para una explicación detallada):

Dimensión Integrativa $=$ Integración - Evitación

Dimensión Distributiva $=$ Dominación - Servilismo

Debido a que el formato de respuesta del ROCI-II es una escala Likert de 1 a 5 , el rango de puntuaciones posible de las dimensiones integrativa y distributiva varía entre -4 y +4 . En la dimensión integrativa una puntuación de +4 representa el esfuerzo de la persona por satisfacer los intereses de ambas partes, y una puntuación de -4 representa un escaso o nulo interés por las necesidades de ambos. En la dimensión distributiva, una puntuación de +4 representa un mayor interés por satisfacer los objetivos personales que los del oponente, mientras que una puntuación de -4 representa un mayor interés por satisfacer las demandas del oponente que los objetivos propios.

En este trabajo, el rango de puntuaciones obtenido para la dimensión integrativa fue de -2.05 a 3.19 , con un valor medio de .61 $(D T=.73)$. Para la dimensión distributiva el rango fue de -2.87 a 3.33 , con una media de $.20(D T=.17)$.

$\mathrm{Al}$ examinar las claves emocionales de la dimensión distributiva, no se ha hallado un modelo significativo, $F(2$,
$437)=.72, n s$. Esto es, no se ha encontrado efectos principales del afecto positivo $(B=.08, S E=.07, n s)$ ni del afecto negativo $(B=.05, S E=.08, n s)$ sobre la dimensión distributiva. En cambio, al analizar si el afecto positivo y el afecto negativo predicen la dimensión integrativa, se han encontrado resultados significativos, $F(2,437)=5.15, p<.01$. En concreto, el afecto positivo $(B=.15, S E=.05, p<.01)$, pero no el afecto negativo $(B=-.07, S E=.06, n s)$, se relaciona con la preocupación por los intereses propios y los intereses de los demás, confirmando los resultados obtenidos en los análisis previos.

\section{Discusión y conclusiones}

El presente trabajo ha sido diseñado para identificar las claves emocionales que operan detrás de la elección de las estrategias de manejo de conflictos interpersonales. Concretamente, se ha examinado si los estados de ánimo positivos y negativos pueden predecir la preferencia por cuatro estrategias de gestión de conflictos, a saber: integración, dominación, evitación y servilismo. A diferencia de estudios previos, que manipulan artificialmente las emociones de los participantes (p.e., Carnevale e Isen, 1986), este trabajo aborda el estado de ánimo que los sujetos experimentan de modo natural. Esto es, se parte del concepto de afecto prenegociación introducido por Barry y Oliver (1996), que representa el conjunto de emociones y afectos que experimenta un persona antes de hacer frente a un conflicto y que está estrechamente ligado a su nivel de afecto disposicional (Watson et al., 1988).

Los resultados de la presente investigación empírica muestran que las estrategias de conflicto están relacionadas con la experiencia emocional, dando apoyo adicional a los hallazgos previos en el campo de la gestión de conflictos y la negociación (p.e., Allred et al., 1997; Baron, 1990; Baron et al., 1990; Carnevale e Isen, 1986; Desivilya y Yagil; 2005; Forgas, 1998; Isen et al., 1987; Kramer et al., 1993; Forgas, 1998; Forgas y Cromer, 2004; Montes et al., 2012; Pillutla y Murnighan, 1996; Rhoades et al., 2001). En concreto, los datos del presente estudio indican que la preferencia por la estrategia integrativa se asocia a la experiencia emocional positiva. En cambio, la preferencia por la estrategia de dominación se acentúa cuando los sujetos experimentan emociones negativas. De este modo, como señala Elfenbein (2007), los sujetos que experimentan alto AP están más centrados (externamente) en la promoción de resultados positivos, mientras que los sujetos que experimentan alto AN están más centrados (internamente) en la prevención de resultados negativos.

Estos hallazgos también confirman que incluso los estados emocionales más leves (en nuestro caso, emociones experimentadas de modo natural fuera de laboratorio) pueden influir sobre el comportamiento de las partes $\mathrm{y}$, por tanto, condicionar los resultados del proceso de gestión de conflictos (p.e., Forgas, 1998).

Además, se ha encontrado que la dominación no sólo está relacionada con el afecto negativo, sino que también está 
ligada a las emociones positivas, confirmando los resultados de estudios previos (p.e., Desivilya y Yagil, 2005). Por su parte, cabe señalar que no se ha podido confirmar la relación entre el estado de ánimo de los sujetos y las estrategias de evitación y servilismo.

En un esfuerzo por profundizar en la relación entre el afecto y el comportamiento de conflicto, se ha examinado la relación entre los estados de ánimo y las dimensiones integrativa y distributiva subyacentes a las estrategias de manejo de conflictos. Los resultados corroboran, nuevamente, que los estados de ánimo positivos se relacionan con el grado en que los sujetos manifiestan interés por sus propios intereses y por los intereses de los demás. Por el contrario, no se ha hallado relación entre el AP o el AN con la dimensión distributiva.

A pesar de que esta línea de investigación no está exenta de críticas (p.e., Hertel y Fiedler, 1994), se puede concluir que las emociones son una fuerza dinámica imprescindible para comprender los fenómenos sociales de colaboración y competición (Obeidi, Hipel, y Kilgour, 2005) y que, a tenor de los resultados expuestos, las emociones son especialmente relevantes para comprender las estrategias y tácticas desplegadas en un conflicto (Barry y Oliver, 1996).

\section{Implicaciones teóricas}

La experiencia emocional se organiza de modo circular en torno a dos dimensiones básicas (Feldman y Russell, 1998; Larsen y Diener, 1992; Russell, 1980; Watson y Tellegen, 1985), a saber: Arousal o nivel de activación y Valencia o tono hedónico. Sin embargo, este hecho no parece tenerse en cuenta en las investigaciones existentes sobre conflicto y negociación, ya que éstas suelen describir sus hallazgos de modo exclusivo en términos de la valencia afectiva (es decir, afecto positivo $v$ s. negativo, estados de ánimo positivos $v s$. negativos, o emociones positivas $v s$. negativas).

En este sentido, los resultados obtenidos en el presente estudio son especialmente relevantes a la hora de dilucidar los mecanismos emocionales que indicen sobre la preferencia por una u otra estrategia de conflicto, ya que ponen de manifiesto que los estados de ánimo experimentados de modo más vívido son los que tienen un mayor impacto sobre el comportamiento de conflicto. Concretamente, de acuerdo con los resultados del presente trabajo, los estados

\section{Referencias}

Allred, K. G., Mallozzi, J.S., Matsui, F. y Raia, C. P. (1997). The influence of anger and compassion on negotiation performance. Organizational Behavior and Human Decision Processes, 70(3), 175-187. Doi: 10.1006/obhd.1997.2705

Andrade, E. B. y Ariely, D. (2009). The enduring impact of transient emotions on decision making, Organizational Behavior and Human Decision Processes, 109(1), 1-8. Doi: 10.1016/j.obhdp.2009.02.003

Baron, R. A. (1990). Environmentally induced positive affect: Its impact on self-efficacy, task performance, negotiation, and conflict. Journal of $A p$ plied Social Psychology, 20(5), 368-384. Doi: 10.1111/j.15591816.1990.tb00417.x de ánimo parecen estar más relacionados con la dimensión de interés propio que con la dimensión de interés por los demás del Dual Concern Model. De hecho, los análisis efectuados muestran que los estados de ánimo -especialmente el afecto positivo-, sólo predicen las estrategias de gestión de conflictos caracterizadas por un elevado interés propio (integración y dominación), mientras que no están relacionadas con las estrategias que comparten un bajo interés propio (evitación y servilismo). Esto puede ser debido a que, como se señaló anteriormente, los estados de ánimo positivos y negativos han sido evaluados mediante la escala PANAS, cuyos adjetivos conjugan tono hedónico (positivo o negativo) y un nivel de activación elevado (Watson et al., 1988). No es de extrañar, por tanto, que las estrategias que implican más actividad para el propio sujeto y que se traducen en un mayor interés propio se puedan predecir a partir de estados de ánimo como, por ejemplo, estar interesado, animado, enérgico, entusiasmado, activo, o tenso, asustado y enojado.

Futuras investigaciones deberían abordar la relación entre el afecto y las estrategias de gestión de conflictos, operativizando las emociones de acuerdo a su nivel de activación (emociones activas $v$ s. pasivas), y su tono hedónico (emociones positivas $v s$. negativas).

\section{Implicaciones para la práctica}

De los resultados de este trabajo se extraen fundamentalmente dos líneas paralelas de intervención para la práctica y el ejercicio profesional. Por una parte, dado que las estrategias de gestión de conflictos representan un modo particular de responder al conflicto que puede ser prescrito y aprendido (Tidd y Friedman, 2002), los programas de formación y entrenamiento en habilidades de manejo de conflictos deberían incorporar una perspectiva socio-afectiva que reconozca, no sólo la importancia de las emociones en la experiencia y el desarrollo del propio conflicto, sino también el papel que éstas tienen en la preferencia por las estrategias de conflicto. Por otra parte, cabe advertir que, dado que el afecto se puede inducir fácilmente (Carnevale e Isen, 1986), gestionar el clima emocional previo a un encuentro de negociación (p.e., promoviendo un ambiente emocional positivo), permitirá desarrollar un proceso negociador más constructivo y colaborador, facilitando la consecución de resultados más eficaces para ambas partes.

Baron, R. A., Fortin, S. P., Frei, R. L., Hauver, L.A. y Shack, M. L. (1990). Reducing organizational conflict: The role of socially-induced positive affect. International Journal of Conflict Management, 1(2), 133-152. Doi: $10.1108 / \mathrm{eb} 022677$

Barry, B. (2008). Negotiator affect: The state of the art (and the science). Group Decision and Negotiation, 17(1), 97-105. Doi: 10.1007/s10726-0079086-6

Barry, B. y Fulmer, I. S. (2004). Methodological challenges in the study of negotiator affect. International Negotiation, 9(3), 485-502. Doi: $10.1163 / 1571806053498797$ 
Barry, B., Fulmer, I. S. y Van Kleef, G. A. (2004). I laughed, I cried, I settled: The role of emotion in negotiation. En M. J. Gelfand y J.M. Brett (Eds.), The handbook of negotiation and culture: Theoretical advances and crosscultural perspectives (pp. 71-94). Palo Alto, CA: Stanford University Press.

Barry, B. y Oliver, R. L. (1996). Affect in dyadic negotiation: A model and propositions. Organizational Behavior and Human Decision Process, 67(2), 127-143. Doi: 10.1006/obhd.1996.0069

Barsade, S. G. y Gibson, D. E. (2007). Why Does Affect Matter in Organizations? Academy of Management Perspectives, 21(1), 36-59. Doi: 10.5465/AMP.2007.24286163

Bazerman, M., Curhan, J., Moore, D. y Valley, K. (2000). Negotiation. Annual Review of Psychology, 51, 279-314. Doi: 10.1146/annurev.psych.51.1.279

Bell, C. y Song, F. (2005). Emotions in the conflict process: An application of the cognitive appraisal model of emotions to conflict management. International Journal of Conflict Management, 16(1), 30-54. Doi: 10.1108/eb022922

Blake, R. R. y Mouton, J. S. (1964). The managerial grid. Houston, TX: Gulf.

Carnevale, P. J. e Isen, A. M. (1986). The influence of positive affect and visual access on the discovery of integrative solutions in bilateral negotiation. Organizational Behavior and Human Decision Processes, 37(1), 1-13. Doi: 10.1006/obhd.1997.2734

Carnevale, P. J. y Lawler, E. J. (1986). Time pressure and the development of integrative agreements in bilateral negotiations. Journal of Conflict Resolution, 30(4), 636-659. Doi: 10.1177/0022002786030004003

Cohen, J., Cohen, P., West, S.G. y Aiken, L.S. (2003). Applied multiple regression/correlation analysis for the behavioural sciences ( ${ }^{\mathrm{a}} \mathrm{Ed}$.). Nueva Jersey: Erlbaum.

De Dreu, C. K. W., Beersma, B., Steinel, W. y Van Kleef, G. A. (2007). The psychology of negotiation: Principles and basic processes. En A.W. Kruglanski y E.T. Higgins (Eds.), Handbook of basic principles in social psychology (2nd ed., pp. 608-629). New York: Guilford.

De Dreu, C. K. W., Evers, A., Beersma, B., Kluwer, E. S. y Nauta, A. (2001). A theory-based measure of conflict management strategies in the workplace. Journal of Organizational Behavior, 22(6), 645-668. Doi: 10.1002/job.107

De Dreu, C. K. W., Giebels, E. y Van de Vliert, E. (1998). Social motives and trust in integrative negotiation: The disruptive effect of punitive capability. Journal of Applied Psychology, 83(3), 408-422. Doi: 10.1037/00219010.83.3.408

Desivilya, H. S. y Yagil, D. (2005). The role of emotions in conflict management: The case of work teams. International Journal of Conflict Management, 16(1), 55-69. Doi: 10.1108/eb022923

Elfenbein, H. A. (2007). Emotion in organizations. En J. P. Walsh y A. P. Brief (Eds.), The Academy of Management Annals (pp. 315-386). London: Routledge.

Feldman, L. y Russell, J. A. (1998). Independence and bipolarity in the structure of current affect. Journal of Personality and Social Psychology, 74(4), 967984. Doi: 10.1037/0022-3514.74.4.967

Forgas, J. P. (1998). On feeling good and getting your way: Mood effects on negotiation cognition and bargaining strategies. Journal of Personality and Social Psychology, 74(3), 565-577. Doi: 10.1006/obhd.2001.2971

Forgas, J. P. y Cromer, M. (2004). On being sad and evasive: Affective influences on verbal communication strategies in conflict situations. Journal of Experimental Social Psychology, 40(4), 511-518. Doi: 10.1016/j.jesp.2003.06.005

Forgas, J. P. y George, J. (2001). Affective influences on judgments and behaviour in organizations: An information processing perspective. Organirational Behavior and Human Decision Processes, 86(1), 3-34. Doi: 10.1006/obhd.2001.2971

Frijda, N. H. (1993). The place of appraisal in emotion. Cognition and Emotion, 7(3/4), 357-387. Doi: 10.1080/02699939308409193

George, D. y Mallery, M. (2003). Using SPSS for Windows step by step: A simple guide and reference. Boston, MA: Allyn y Bacon.

Hall, J. (1969). Conflict management survey: A survey of one's characteristic reactions to and handling of conflicts between bimself and others. Monroe, TX: Telemetrics International.

Hertel, G. y Fiedler, K. (1994). Affective and cognitive influences in a social dilemma game. European Journal of Social Psychology, 24(1), 131-145. Doi: 10.1002 / ejsp.2420240110
Hocker, J. L. y Wilmot, W.W. (1998). Interpersonal conflict (5th Ed.). Madison, WI: Brown and Benchmark.

Isen, A. M., Daubman, K. A. y Nowicki, G. P. (1987). Positive affect facilitates creative problem solving. Journal of Personality and Social Psychology, 52(6), 1122-1131. Doi: 10.1037/0022-3514.52.6.1122

Kramer, R. M., Newton, E. y Pommerenke, P. L. (1993). Self-enhancement biases and negotiator judgment: Effects of self-esteem and mood. $\mathrm{Or}$ ganizational Behavior and Human Decision Processes, 56(1), 110-133. Doi: 10.1006/obhd.1993.1047

Montes, C., Serrano, G. y Rodríguez, D. (2010). Impacto de las motivaciones subyacentes en la elección de las estrategias de conflicto. Boletín de Psicología, 100(4), 55-69.

Montes, C., Rodríguez, D. y Serrano, G. (2012). Affective choice of conflict management styles. International Journal of Conflict Management, 23(1), 6-18. Doi: 10.1108/10444061211199304

Morris, M. W. y Keltner, D. (2000). How emotions work: The social functions of emotional expression in negotiations. Research in Organizational Behavior, 22, 1-50. Doi: 10.1016/S0191-3085(00)22002-9

Munduate, L., Ganaza, J. y Alcaide, M. (1993). Estilos de gestión del conflicto interpersonal en las organizaciones. Revista de Psicología Social, 8(1), 47-

Nair, N. (2008). Towards understanding the role of emotions in conflict: A review and future directions. International Journal of Conflict Management, 19(4), 359-381. Doi: 10.1108/10444060810909301

Nunnally, J. C. (1978). Psychometric Theory. New York, NY: McGraw-Hill.

Obeidi, A., Hipel, K. W. y Kilgour, D. M. (2005). The role of emotions in envisioning outcomes in conflict analysis. Group Decision and Negotiation, 14(6), 481-500. Doi: 10.1007/s10726-005-9004-8

Pillutla, M. M. y Murnighan, J. K. (1996). Unfairness, anger, and spite: Emotional rejections of ultimatum offers. Organizational Behavior and Human Decision Processes, 68(3), 208-224. Doi: 10.1006/obhd.1996.0100

Pruitt, D. G. (1983). Strategic choice in negotiation. American Behavioral Scientist, 27(2), 167-194. Doi: 10.1177/000276483027002005

Pruitt, D. G. y Carnevale, P. J. (1993). Negotiation in social conflict. Buckingham: Open University Press.

Pruitt, D. G. y Lewis, S. A. (1975). Development of integrative solutions in bilateral negotiation. Journal of Personality and Social Psychology, 31(4), 621633. Doi: 10.1037/0022-3514.31.4.621

Pruitt, D. G. y Rubin, J. Z. (1986). Social Conflict: Escalation, Stalemate, and Settlement. New York: Random House.

Putnam, L. L. y Poole, M. S. (1987). Conflict and negotiation. En Jablin, F. M., Putnam, L. L., Roberts, K. H. y Porter, L. W. (Eds.), Handbook of organizational communication: An interdisciplinary perspective (pp. 549-599). Newbury Park, CA: Sage.

Rahim, M. A. (1983). A measure of styles of handling interpersonal conflict. Academy of Management Journal, 26(2), 368-376. Doi: 10.2307/255985

Rahim, M. A. (1986). Managing Conflicts in Organizations. New York: Praeger.

Rahim, M. A. (2002). Toward a Theory of Managing Organizational Conflict. International Journal of Conflict Management, 13(3), 206-235. Doi: 10.1108/eb022874

Rahim, M. A. y Bonoma, T. V. (1979). Managing organizational conflict: A model for diagnosis and intervention. Psychological Reports, 44(3), 13231344. Doi: $10.2466 /$ pr0.1979.44.3c.1323

Rahim, M. A. y Magner, N. R. (1995). Confirmatory factor analysis of the styles of handling interpersonal conflict: First-order factor model and its invariance across groups. Journal of Applied Psychology, 80(1), 122-132. Doi: 10.1037/0021-9010.80.1.122

Rhoades, J. A., Arnold, J. y Jay, C. (2001). The role of affective traits and affective states in disputants' motivation and behavior during episodes of organizational conflict. Journal of Organizational Behavior, 22(3), 329-345. Doi: 10.1002/job.72

Sandín, B., Chorot, P., Lostao, L., Joiner, T. E., Santed, M. y Valiente, R. (1999). Escalas de Afecto Positivo y Negativo: Validación factorial y convergencia transcultural. Psicothema, 11(1), 37-51.

Tidd, S. y Friedman, R. (2002). Conflict style and coping with role-conflict: An extension of the uncertainty model of work stress. International Journal of Conflict Management, 13(3), 236-257. Doi: 10.1108/eb022875

Thomas, K.W. (1976). Conflict and conflict management. En Dunnette, M. D. (Ed.), Handbook of Industrial and Organizational Psychology (pp. 889-935). Palo Alto, CA: Consulting Psychologists Press. 
Van de Vliert, E. y Hordijk, J. W. (1989). A theoretical position of compromising among other styles of conflict management. Journal of Social Psychology, 129(5), 681-690. Doi: 10.1080/00224545.1989.9713785

Van Kleef, G. A., De Dreu, C. K. W. y Manstead, A. S. R. (2004a). The interpersonal effects of anger and happiness in negotiations. Journal of Personality and Social Psychology, 86(1), 57-76. Doi: 10.1037/00223514.86.1.57

Van Kleef, G. A., De Dreu, C. K. W. y Manstead, A. S. R. (2004b). The interpersonal effects of emotions in negotiations: A motivated information processing approach. Journal of Personality and Social Psychology, 87(4), 510-518. Doi: 10.1037/0022-3514.87.4.510

Van Kleef, G. A., De Dreu, C. K. W., Pietroni, D. y Manstead, A. S. R. (2006). Power and emotion in negotiation: Power moderates the effect of anger and happiness on concession making. European Journal of Social Psychology, 36(4), 557-581. Doi: 10.1002/ejsp.320
Watson, D., Clark, L. A. y Tellegen, A. (1988). Development and validation of brief measures of positive and negative affect: The PANAS scales. Journal of Personality and social Psychology, 54(6), 1063-1070. Doi: 10.1037/0022-3514.54.6.1063

Watson, D. y Tellegen, A. (1985). Toward a consensual structure of mood. Psychological Bulletin, 98(2), 219-235. Doi: 10.1037/0033-2909.98.2.219

Weingart, L. R., Bear, J. y Todorova, G. (2009). Excited to disagree? A Study of Conflict and Emotions. Comunicación presentada en el 22nd Annual International Association of Conflict Management Conference (IACM), 1518 de Junio, Kyoto, Japan.

Weingart, L. R., Hyder, E. B. y Prietula, M. J. (1996). Knowledge matters: The effect of tactical descriptions on negotiation behavior and outcome. Journal of Personality and Social Psychology, 70(6), 1205-1217. Doi: 10.1037/0022-3514.70.6.1205

(Artículo recibido: 08-09-2011; revisado: 23-01-2013; aceptado: 23-01-2013) 